\title{
Avoiding Aesthetic Drawbacks and Stigmata in Dorsal Line Preservation Rhinoplasty
}

\author{
José Carlos Neves, $\mathrm{MD}^{1}$ Diego Arancibia-Tagle, $\mathrm{MD}^{1,2}$ \\ ${ }^{1}$ Department of Facial Plastic Surgery, MyFace Clinics, Lisboa, \\ Portugal \\ 2 Department of Otolaryngology and Head and Neck Surgery, Hospital \\ Universitari Son Espases, Palma de Mallorca, Baleares, Spain \\ Address for correspondence Diego Arancibia-Tagle, MD, Department \\ of Otolaryngology and Head and Neck Surgery, Hospital Universitari \\ Son Espases, 07120 Palma de Mallorca, Baleares, Spain \\ (e-mail: arancibiadiego@gmail.com).
}

Facial Plast Surg 2021;37:65-75.

\begin{abstract}
Keywords

- rhinoplasty

- preservation

- Tetris concept

- segmental preservation

For over a century, discussion on the management of nasal dorsum has been a struggle between the techniques of resection and preservation. This is because, each technique has its advantages and disadvantages, with the Joseph technique dominating over the past 30 years despite its surgical stigmas. The dorsum preservation techniques offers a good option for the treatment of nasal hump but like resective techniques it has its drawbacks. This may be the reason why preservation techniques withered but are now making a resurgence. The aim of this article is to describe how to avoid the aesthetic drawbacks of this technique depending on the selected approach.
\end{abstract}

For many years, a number of surgeons have pursued the goal of modifying the aesthetics of the nose while preserving the dorsal structure of the nasal pyramid. Starting with Goodale ${ }^{1}$ in the 19th century, the sum of new ideas during the next century was insufficient to revolutionize the mainstream world of rhinoplasty. Lothrop, ${ }^{2}$ Cottle, ${ }^{3,4}$ Huizing, ${ }^{5}$ and $\mathrm{Gola}^{6-8}$ are all examples of those who contributed tremendously to the development of the concept.

Traditionally associated with closed approach rhinoplasty, the new era of the open approach and the excitement it provoked around the world was an obstacle, while in most cases and for many years conservative maneuvers provoked little interest due to being visually unexciting. Knights like Wilson Dewes, Fausto López-Infante, and Yves Saban kept the philosophy alive and inspired many surgeons to start a new chapter.

Like any other surgical technique, dorsal conservative rhinoplasty has its indications and limitations. In this article, we focus on our personal strategies to avoid some of the drawbacks and stigmata of the dorsal line preservation rhinoplasty.

\section{Common Drawbacks in Dorsal Line Preservation Rhinoplasty}

Even though for some surgeons deprojectioning the nasal profile without touching the dorsal line structure was a conceptual revolution, for others, living in their natural habitat of preservation, the challenge was achieving better results in a predictable and consistent way. ${ }^{8-20}$

At the start, the excitement of performing the push-down maneuver (the deprojection maneuver) revealed some fragilities of the technique when not appropriately executed, resulting in very low radix or entire nasal dorsum, with residual dorsal hump or supratip saddling. It is essential to understand the mechanical process of the technique in a tridimensional fashion and choose the right patient to achieve the best result.

Aesthetic drawbacks and stigmata seen in dorsal line preservation rhinoplasty, both in profile and frontal views, as well as functional issues are listed below and discussed (-Table 1).

\section{Profile Drawbacks}

\section{Residual Hump or Hump Recurrence}

The ideal scenario for a pyramid push-down/let-down maneuver is a high flat tension nose. When we face a convex dorsal profile that needs to be flattened, several considerations need to be taken into account to avoid a residual hump or other stigmata postoperatively.

To some extent the definition of residual hump depends on the eye of the beholder. Sometimes results are shown and
Issue Theme Preservation Rhinoplasty: An Update; Guest Editor: Jose Carlos Neves, MD (c) 2021. Thieme. All rights reserved. Thieme Medical Publishers, Inc., 333 Seventh Avenue, 18th Floor, New York, NY 10001, USA 
Table 1 Drawbacks and stigmata

\begin{tabular}{|l|}
\hline Profile view drawbacks and stigmata \\
\hline Hump recurrence \\
\hline Radix step \\
\hline Low nasal radix and dorsum \\
\hline Supratip saddling \\
\hline Frontal view drawbacks \\
\hline Pyramid lateralization \\
\hline Pyramid broadening \\
\hline Functional impairment \\
\hline Blockage associated with push-down (bone impaction) \\
\hline Blockage associated with LKA disarticulation \\
\hline
\end{tabular}

Abbreviation: LKA, lateral Keystone area.

described as having no hump where it is possible to see a residual convexity and based on that some surgeons admit to $<5 \%$ of residual hump while others $15 \% .^{21-23}$ Though one may regard a small hump as natural, it is important to achieve a result planned at preoperative consultation, flat or slightly convex.

In smaller convexities, pushing the pyramid down and hiding a possible radix step below the thick-soft tissues may be a good strategy for producing a flattened profile. We prefer to avoid radix steps, except when the radix is high and bringing it down is part of the surgical strategy. So, routinely we employ additional maneuvers that philosophically question whether we are actually performing real preservation surgery, once we disrupt structures in the foundation of the nasal pyramid.
We consider three aspects to obtain an ideal profile: (1) accurate and predictable deprojection; (2) dorsal line flattening movement; (3) stability of the final position avoiding relapses (-Fig. $\mathbf{1}$ ).

\section{Let-Down Technique}

While the lateral wall can be approached using the pushdown technique (PDO) or the let-down technique (LDO) 24,25 (-Fig. 2A, B), we prefer the LDO since it allows a good pyramid mobilization avoiding bone impaction into the nasal cavity and consequently the benefits of not impinging on nasal airway.

The precision of the wedge resection in LDO has no impact on pyramid stabilization or the final profile position, as the septal wall is the guiding structure dictating the final result. Even if we excise a wedge of bone matching the exact amount of dorsal height deprojection, the two borders of bone are not in contact as the remaining bony pyramid is narrower than the basal bony structure, with the possible contact happening exclusively in the cephalic end. Any gap in the bony continuity left after the pyramid is adjusted to the new position will be filled by neoosteogenesis, because of the periosteal preservation (-Fig. 3).

\section{Splitting the Three Walls}

In low and intermediate strip approaches, the septal wall ideally must be split at the level of the most prominent point of the hump, generally caudal to the rhinion (almost always is septal cartilage that we have to resect), in order to create the necessary movement to correct the convex profile. To be effective when stretching the dorsal convexity, the mid wall should have two pillars (at caudal and a cephalic end of the

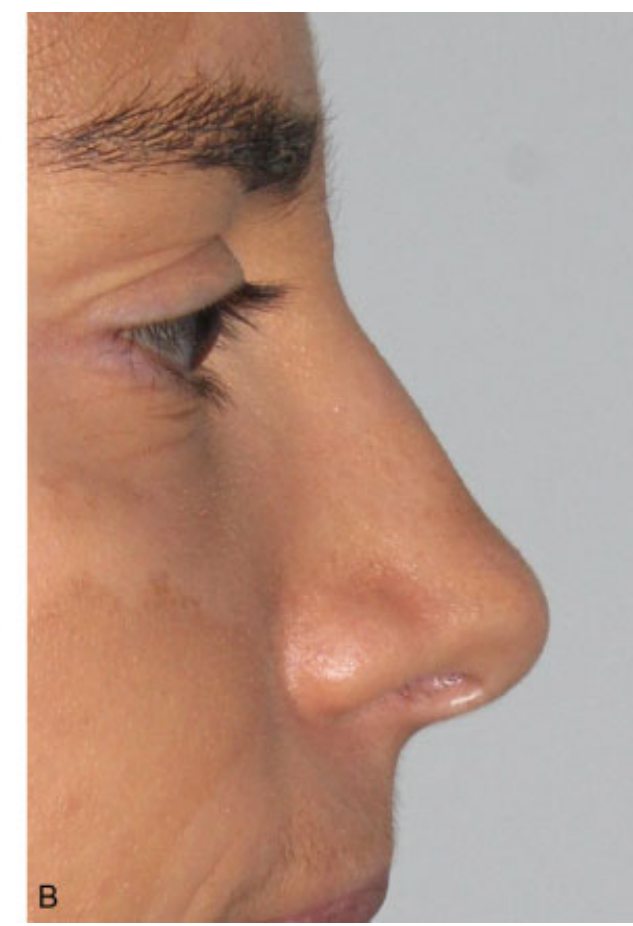

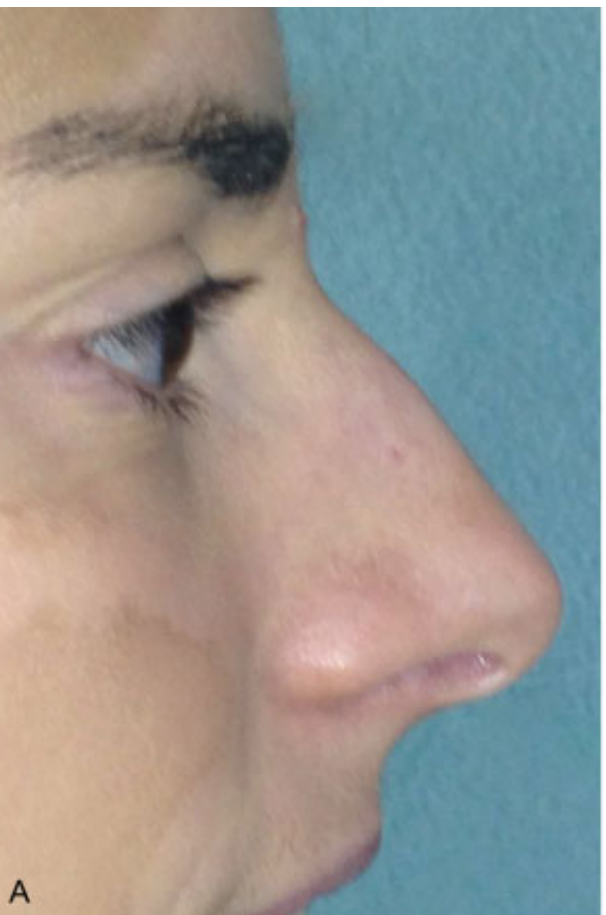

Fig. 1 (A, B) A low strip push-down approach was performed. A residual hump and a minor supratip saddling. 

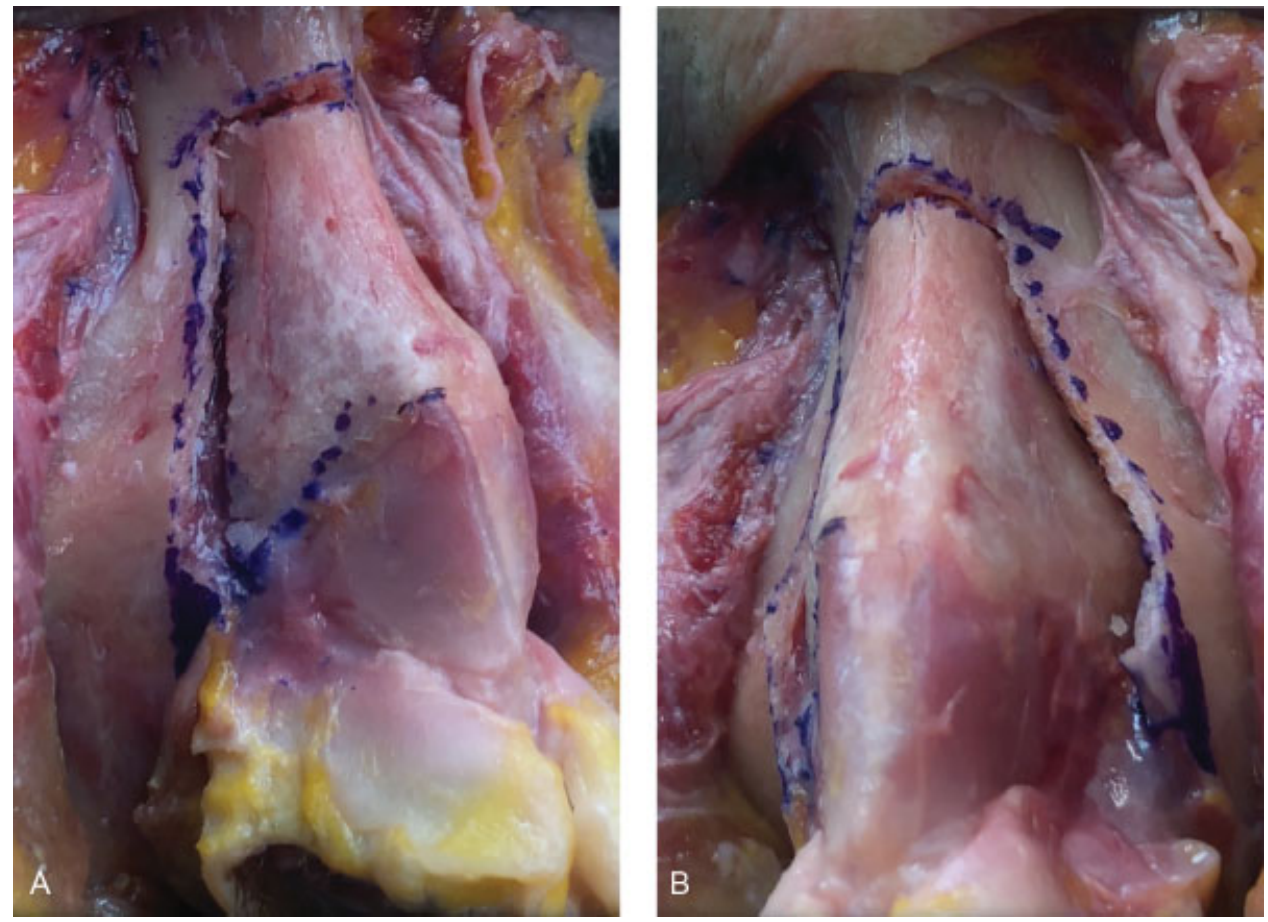

Fig. 2 Cadaveric study showing a let-down technique (A) at the right side (with no bone impaction into the nasal cavity) and a push-down technique (B) at the right side (where the impaction is visible).

curve) supporting the forces imposed in between them, over the splitting point. It works like the splits (-Fig. 4).

The lateral wall should follow the same concept. In most of our cases we perform the lateral $\mathrm{K}$ stone area (LKA) disarticulation (-Figs. 3 and $\mathbf{5}$ ), which creates a lateral split and allows for a sliding movement of the cartilaginous structure in an anterior and caudal vector. ${ }^{26-28}$ The more the distance from the dorsal line the more the limitation in the movements that the pyriform ligament and upper lateral cartilage (ULC) lateral bony wall cause. The lateral wall will work as a
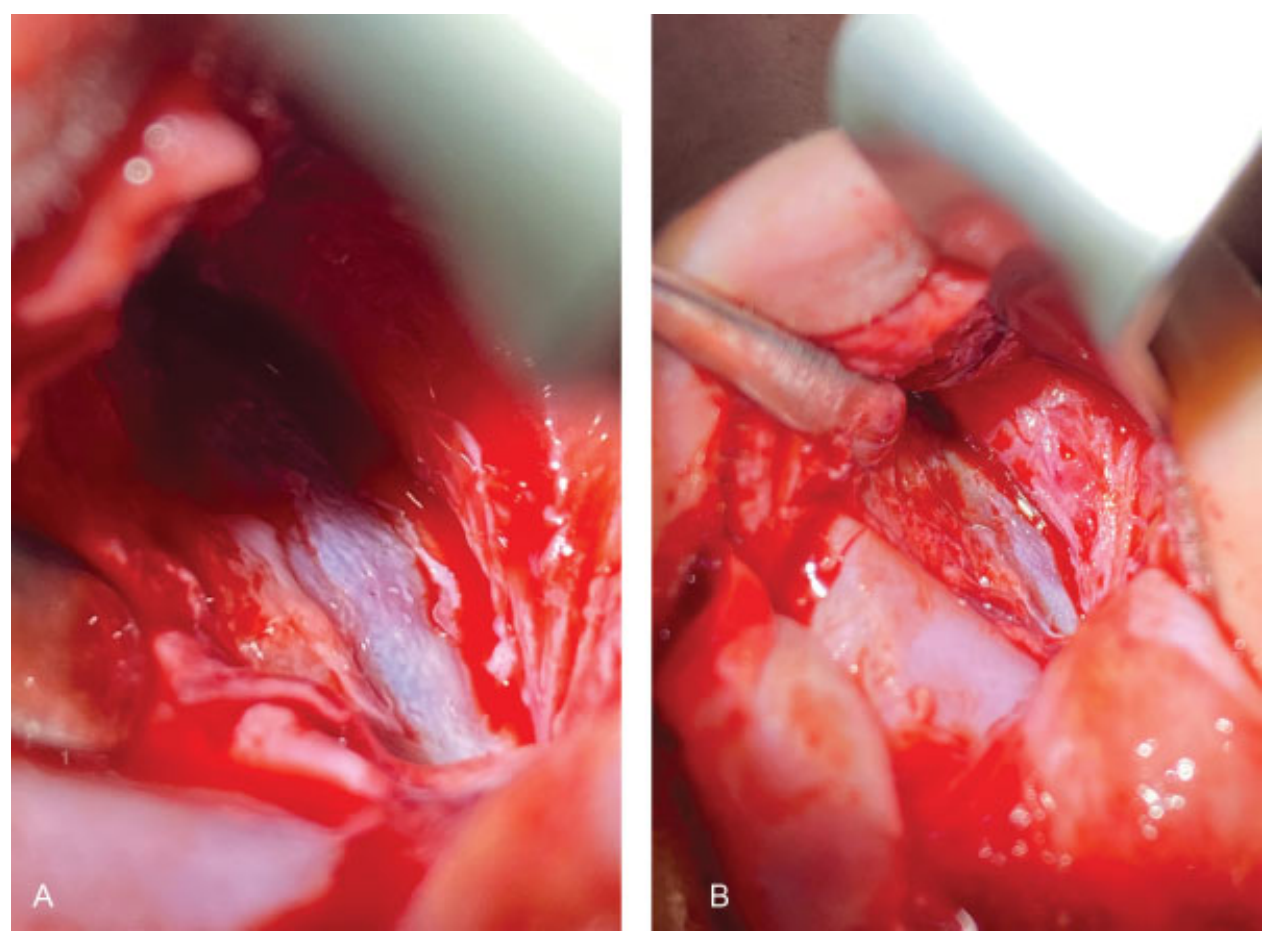

Fig. 3 One-year postoperative revision case; a let down technique was performed. (A) New bone in the gap created is seen, thinner and whiter. (B) An LKA disarticulation was performed in the previous surgery. Note the normal continuity and stability in between the UL and the bony wall. LKA, lateral Keystone area. 


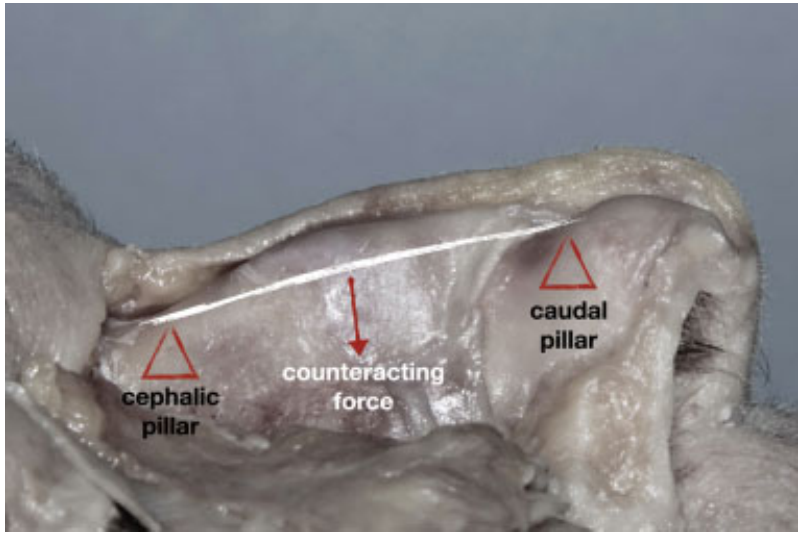

Fig. 4 Two stable pillars must be preserved, one cephalic at the radix area, another one caudal at the supratip region. A force that counteracts the spring effect responsible for hump recurrency is paramount to predict accuracy and stability.

facilitator for defining the final dorsal profile that follows the septal work.

\section{Subrhinion Stabilization}

The spring effect has its maximum force vector at the highest point of the hump. To counter this phenomenon, we preserve a piece of cartilage attached to the pyramid below the most prominent point of the hump that will be anchored with PDS sutures to a stable basal segment of the septum. That means we are performing an intermediate approach or eventually a partial intermediate approach, as in the Tetris concept ${ }^{29,30}$ (- Fig. 6).

In the low approach the probability of relapsing and having a residual hump is greater. All the mobile pyramid is stabilized to the anterior nasal spine region. A stretching effect of the hump is produced by the caudal and anterior rotational movement that flattens the hump. However, the spring effect is not directly counterbalanced. Even if it is not

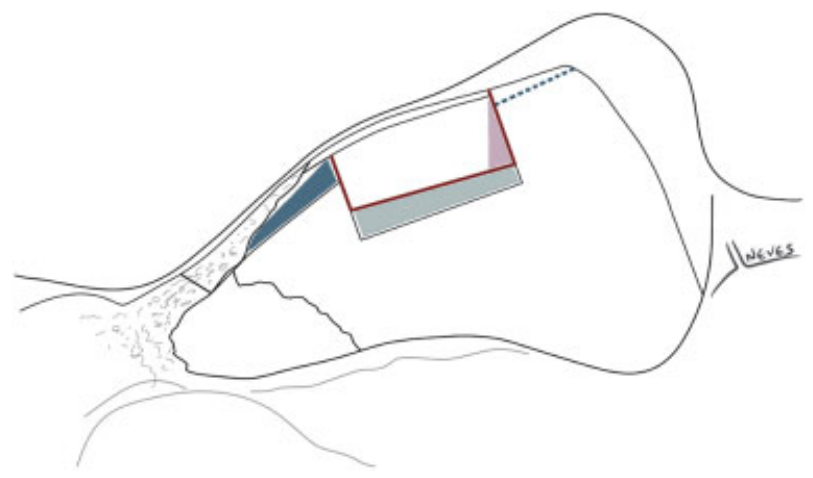

Fig. 6 The Tetris Concept. A 5 to $8 \mathrm{~mm}$ height block is designed in between the WASA and the dorsal hump most prominent point (red line); a trapezoid figure is drawn below the block, it represents the amount of hump to be reduced (gray trapezoid); a triangular figure is drawn below the bone pyramid, from the block till the lateral wall transverse osteotomy level to facilitate the push-down movement (blue triangle); to avoid overlapping the caudal aspect of the Tetris block with the natural caudal septal strut we trim a triangular portion of the block cartilage (purple triangle); to adjust the new dorsal profile level a trimming of the anterior border of the caudal septal strut must be performed (blue dots). WASA, area between the cuadal portion of the upper lateral cartilage and the anterior septal angle.

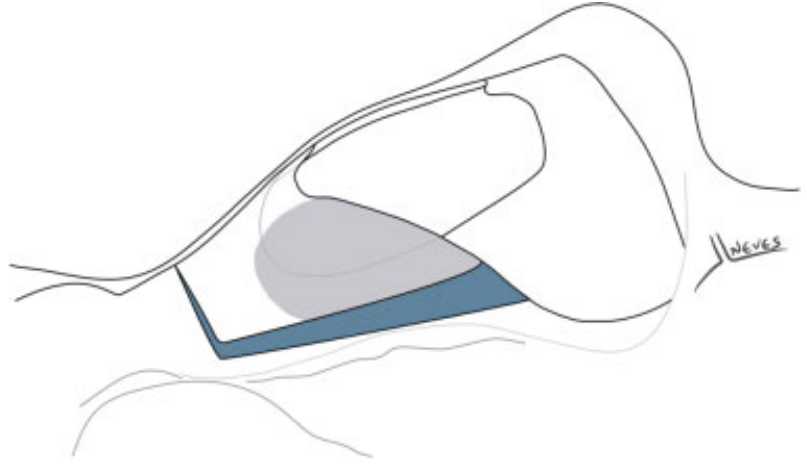

Fig. 5 Let-down technique (blue). The gray shadow represents the periosteal elevation area after releasing the pyriform ligament from the pyriform aperture.

possible to be as effective as the parallel opposite sutures previously described, we use two or three oblique sutures to the spring effect vector to achieve stabilization of the cartilaginous vault ( - Fig. 7 ).

In the high approach a transdorsal suture can be placed, passing over the cartilaginous pyramid and stabilizing it to the basal septum.

\section{Reshaping the Residual Bony Hump}

In dorsal preservation rhinoplasty the analysis of the osseous upper third is paramount. The concept of $S$-shaped and $V$ shaped nasal bones introduced by Lazovic et $\mathrm{al}^{30}$ is being discussed as a guide for the best indications for full dorsal preservation techniques, with the $V$ shape being the best scenario since it will produce a smooth transition to the dorsal cartilaginous surface.

The $S$-shaped nasal bones can promote the appearance of an osseous residual hump that represents a potential stigma of the dorsal conservative procedures. After the deprojection maneuver is performed the appearance of this residual hump may be unnoticed during surgery, therefore refinement maneuvers may have to be considered.

Depending on the approach rasps, burs or piezotome may be used. We prefer cylindric burrs to create smooth surfaces and transitions ( $\boldsymbol{- \text { Fig. }}$ 8). The bony cap will be reshaped to the desired level obtaining the ideal profile line, as well as the lateral walls and the nasofacial groove.

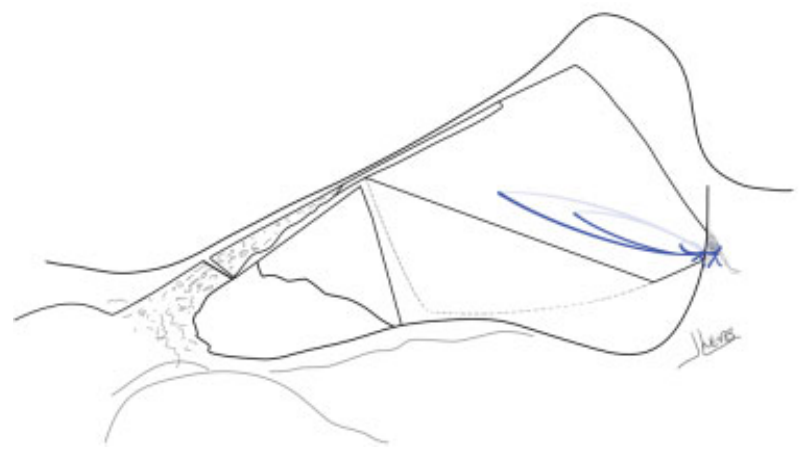

Fig. 7 In a low approach two or three oblique sutures to the spring effect vector are placed to achieve stabilization of the final profile line avoiding recurrences of the hump. 

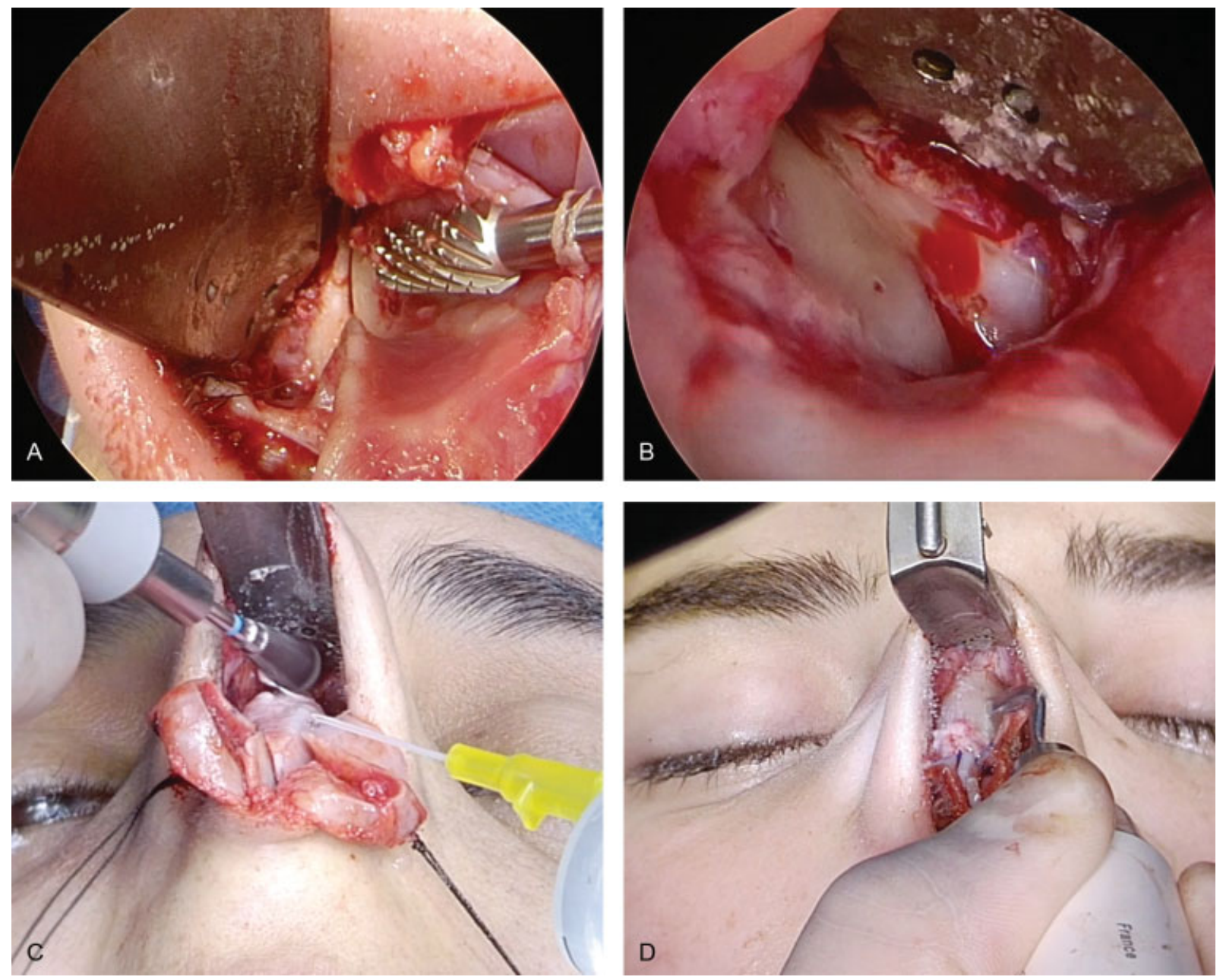

Fig. 8 Refinements in dorsal preservation rhinoplasty. (A) A step at the right nasofacial groove can be seen; a cylindrical burr will be used; (B) Smooth transition at the left nasofacial groove after it has been corrected; (C) Sculpting S-shaped nasal bones; (D) Paramedian osteotomies with an ultrasound device to narrow the bony vault.

\section{Reshaping the Residual Cartilaginous Hump}

The cartilaginous hump can show an intrinsic convexity exhibiting: (1) a residual localized cartilaginous hump (caudal hump greater than the original) and (2) depression at the caudal end of the cartilaginous profile (-Fig. 9).

Controlling the convex arch of the cartilaginous line follows the same principles as used on the septum to control the nasal hump. The cartilaginous septum is split midway of the arch to achieve flatness. This can be done by splitting the quadrangular cartilage into a strategic line. The segmental preservation concept considers the possibility of splitting the Tetris block (-Fig. 10) and so designing a flattened or eventual concave curve. Moreover, by preserving a natural caudal septal strut (in between the anterior nasal septal angle and $\mathrm{W}$ point) it allows us to precisely design the supratip area and avoid eventual saddling.

\section{Radix Position Control}

\section{Periosteum Dissection}

Subperiosteal dissection is the best way to address the upper third of the nose. However, in dorsal preservation rhinoplasty the soft tissues over the radix may be left untouched, completely or partially thus acting as a tent to support an eventual descent of the pyramid at the level of the transverse osteotomies.

\section{Transverse Osteotomies}

Location of transverse osteotomies is crucial. From the level of the medial canthal tendon a line is marked that goes superiorly in an oblique fashion reaching the radix in a more cephalic position, where the radix is deepest from skin surface. This serves to camouflage step deformity should it occur (-Fig. 11).

Routinely, two lateral percutaneous osteotomies follow the drawn line and leave a fragment of bone in the midpoint to facilitate the greenstick fracture and spare a periosteal stripe. If a midline osteotomy is needed (best avoided in most cases), the osteotome must be placed obliquely to obtain two oblique line fractures that support the free pyramid and protect it from collapse.

\section{Septal Wall Work}

With the pyramid free, the support for the bony pyramid entirely comes from the septum. The lateral wall in LDO acts as a facilitator. At this point, the convex pyramidal arch is 

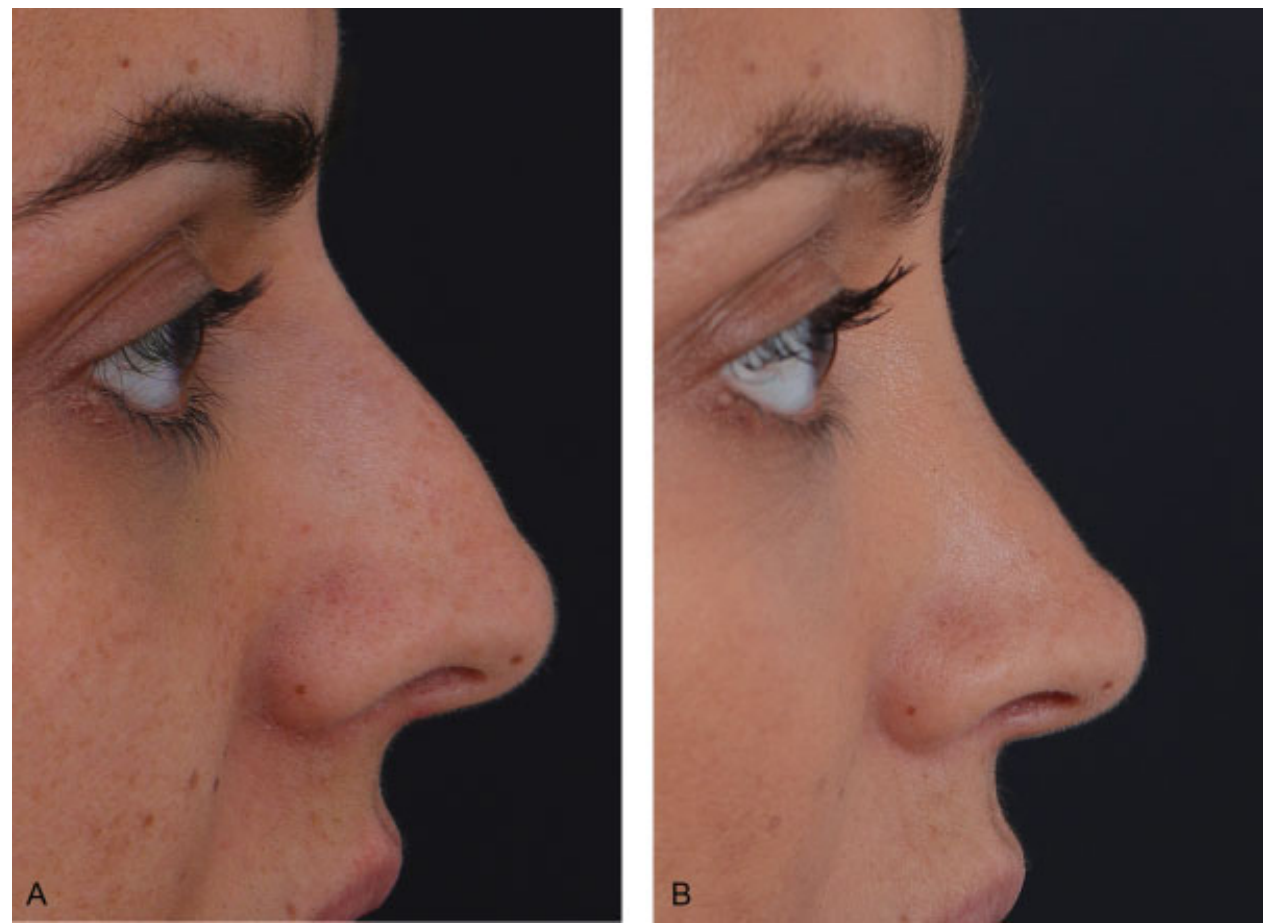

Fig. 9 (A, B) A residual cartilaginous convexity can be seen postoperatively. A full intermediate approach was used with subrhinion stabilization. A cartilaginous split would have helped flattening the cartilaginous profile.

supported by at least two stable pillars, one at the radix, caudal to the transverse osteotomies (cephalic pillar), the other at septal angle (-Fig. 4).

Depending on how the septal segment below the bony vault is addressed the radix keeps its original position, goes up or down. It has both cartilaginous element (the quadrangular cartilage) and osseous one (the perpendicular ethmoidal plate). In most cases, the cartilaginous component is predominant.

Preserving a piece of perpendicular plate below the bony dorsum (extending caudal to the transverse osteotomies) gives the necessary nasal pyramid support and avoids collapse and radix step.

In most cases, cartilaginous septum is trimmed precisely with sharp, slightly curved scissors, to avoid cartilage shearing. Large instruments like rongeurs are best avoided. Tech-

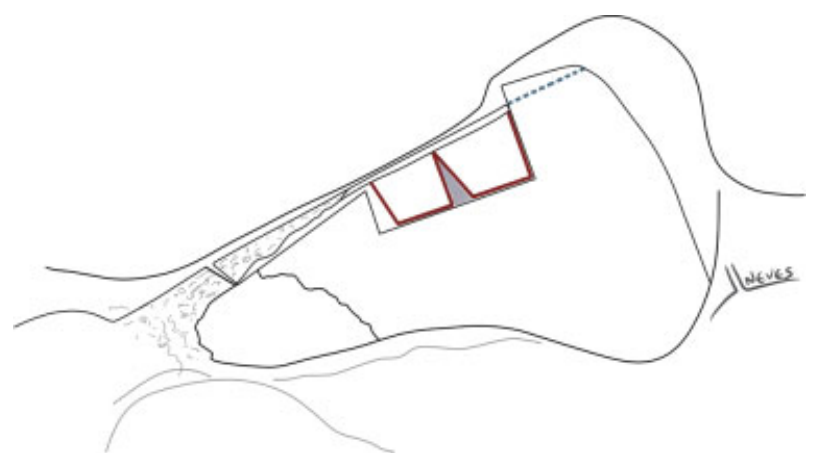

Fig. 10 The split Tetris concept. Splitting the Tetris block (red lines) allows the cartilaginous segment to flatten or eventually to become concave. The wider the gray triangle the more concave this segment will be. nically, the scissors are inserted convex side up, tangentially to the inner surface of the nasal vault from the splitting point to the transverse osteotomy point. This will free the nasal pyramid from the septum. Then, small triangles are excised using the scissors with concavity looking up and adjusting the ideal profile. At this point, different options include:

1. Keeping the radix at the same level: The exact amount of septal excess is resected and the pyramid rests completely on the septum or only a cephalic stable portion of PEP is

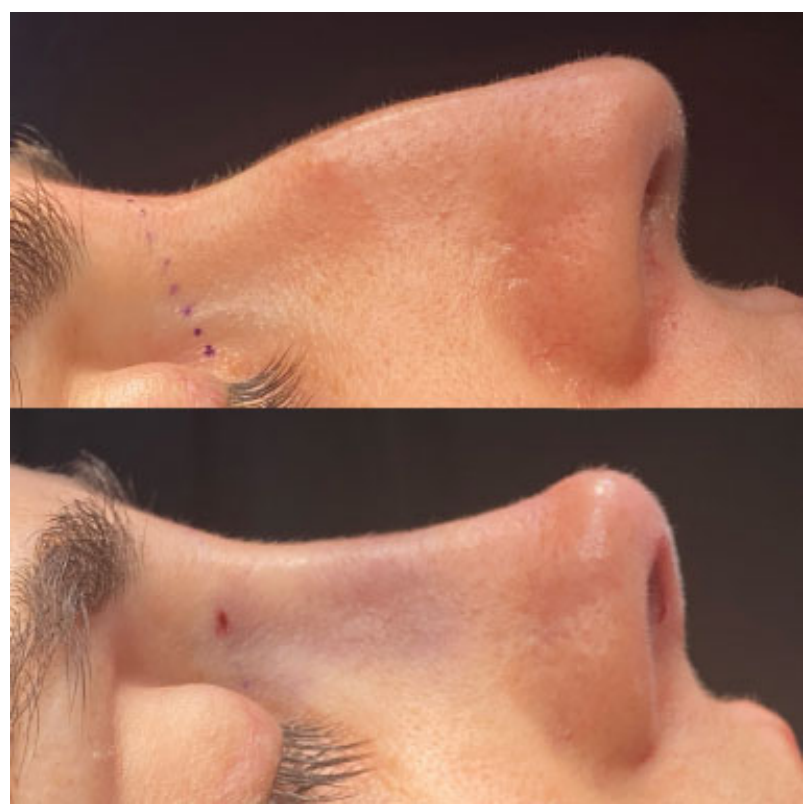

Fig. 11 Anterior and cephalic line to perform a transcutaneous transverse osteotomy. 

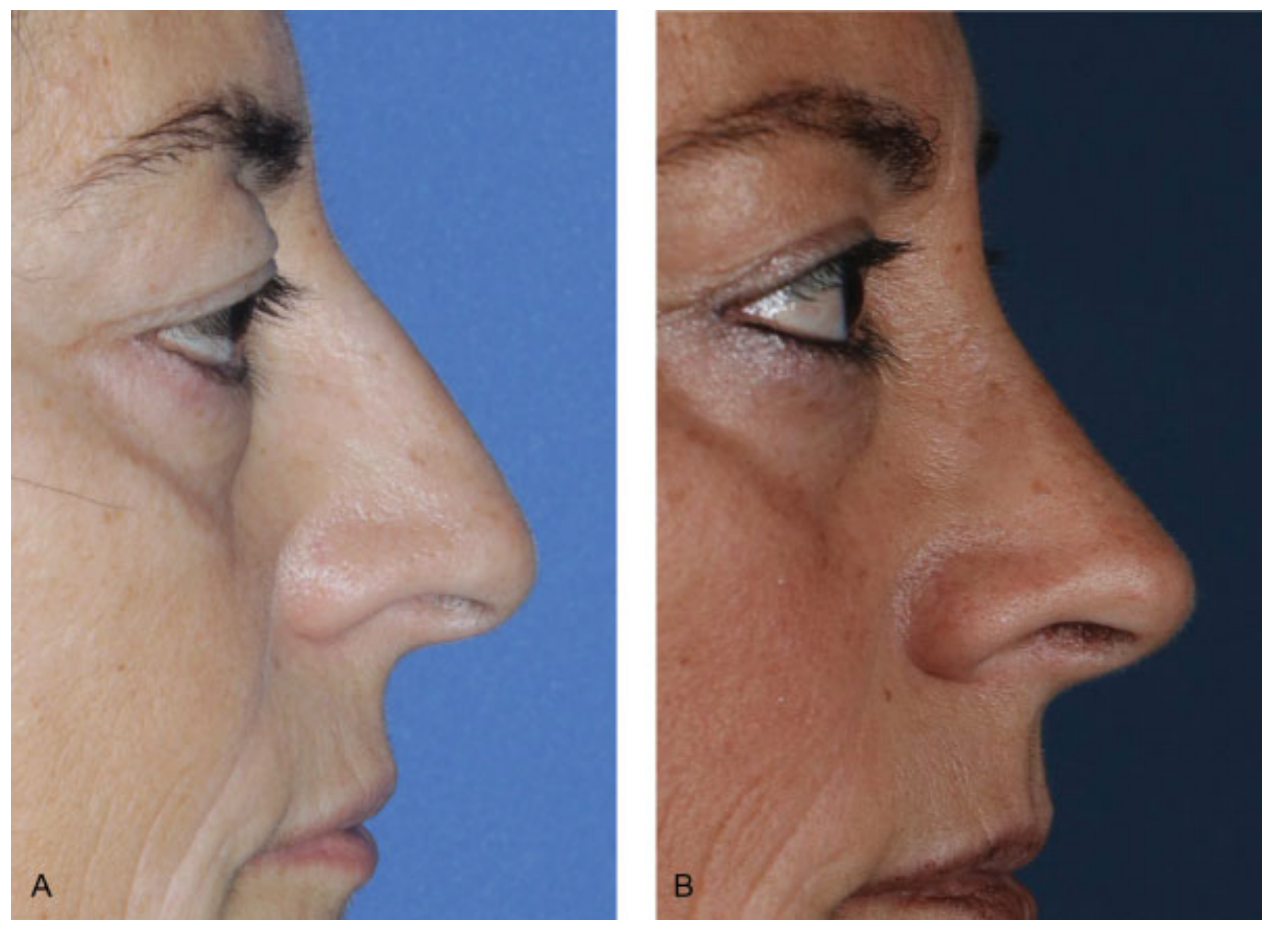

Fig. 12 Let down technique with perpendicular ethomoidal plate control bringing the radix down to a pleasant level.

preserved working as a true pillar for the pyramid stability, allowing extensive septoplasty.

2. Creating a step-down: As already mentioned, the transverse osteotomies can be performed strategically where an eventual step-down is camouflaged by the overlying thick-soft tissues, promoting a bony step-down not visible in profile. When the radix is high and the nose appears to start at eyebrow level, it is possible to create a lower starting point by bringing the radix area down. The septal supportive point of the pyramid is resected incrementally until the profile reaches the desired level (-Fig. 12). This is a delicate maneuver that requires an accurate cut of the septum, especially at perpendicular ethmoidal plate. If resected excessively free pyramid can collapse with disastrous results that must be compensated with grafts (-Fig. 13).

3. Creating a step-up: Preservation technique is not suited for low radix patients. However, when radix position is controlled as already explained, one can achieve to lift the radix using step-up technique: when approaching the septum, we define a pivotal point where the pyramid remains at the same position. Caudal to it the pyramid is
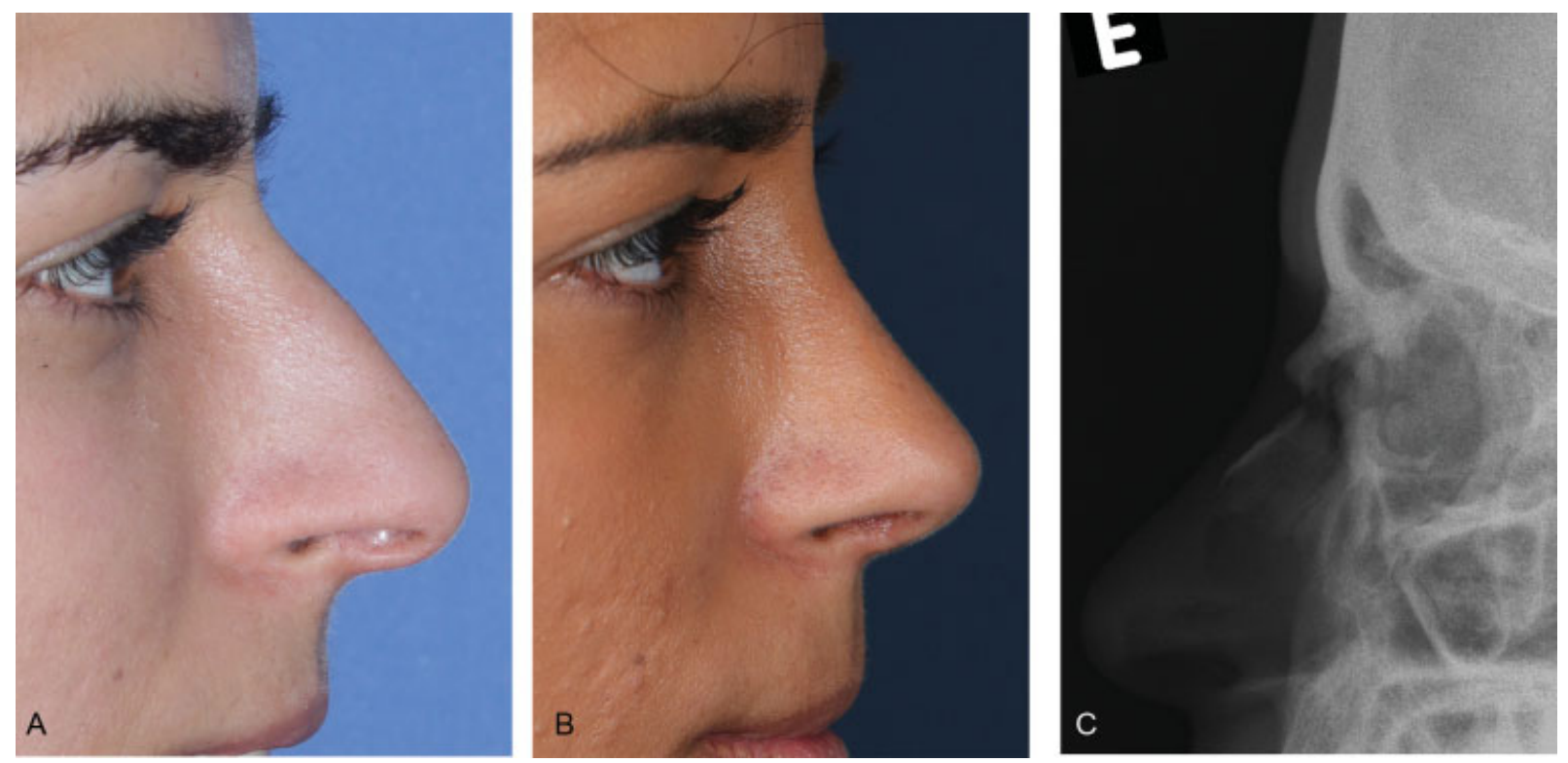

Fig. 13 (A, B) Let down technique with loss of perpendicular ethomoidal plate control creating a low radix of the nose, that were partially compensated with grafts. (C) The Rx image shows the loss of control of the patient's pyramid. 

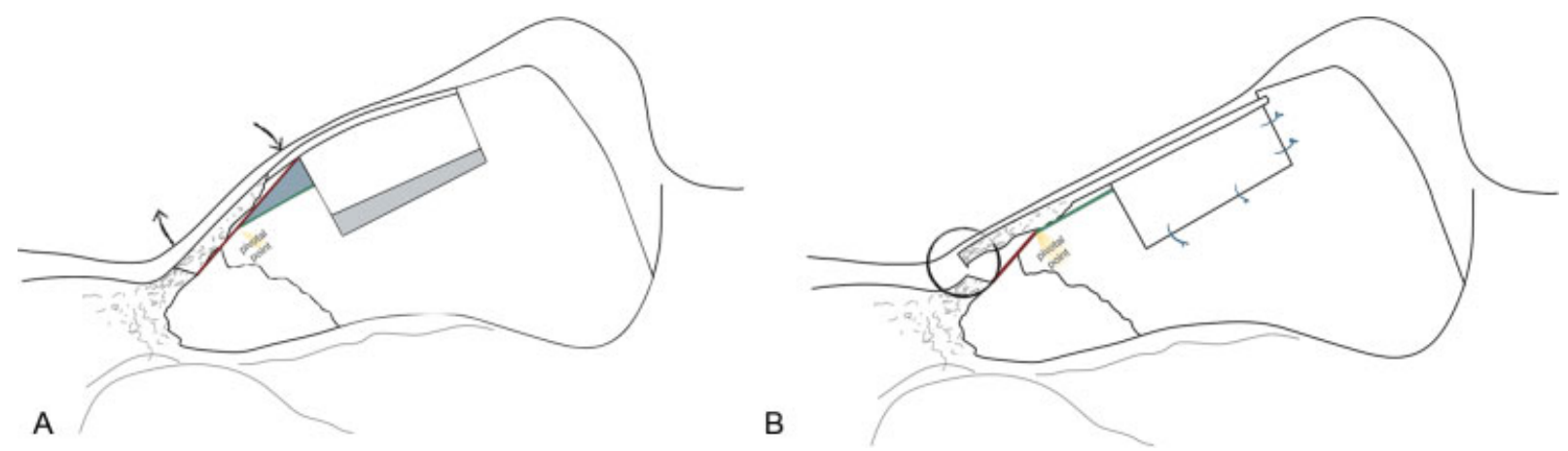

Fig. 14 (A) A pivotal point where the pyramid remains at the same position is designed. (B) Caudal to the pivotal point the pyramid is pushed down; cephalic to it the pyramid goes up, creating the desired radix step-up.

pushed down and cephalic to it the pyramid goes up, creating the desired radix step-up. After defining the pivotal point, a predefined triangular piece of septum is trimmed caudal to the pivot. This triangular space allows for the deprojection maneuver, and the free pyramid cephalic to the pivot goes up (-Figs. 14 and 15).

\section{Supratip Position Control}

The supratip saddling is a common drawback and stigma especially in low approach preservation rhinoplasty. The main reasons for this are: (1) inability to correct the dorsal convexity, leading to a profile that curves to a low supratip area, (2) poor control of the septal height when resecting septal cartilage excess, (3) poor fixation of the new position of the septum to the anterior nasal spine. Based on the above factors, supratip position should be defined carefully.

Supratip over resection is avoided in the high strip ${ }^{21}$ and the partial intermediate approach by sparing a natural caudal strut that can be trimmed as desired. This also aids in designing precise profile of this segment.

In the low approach, an excess of septal resection at the supratip line must be avoided to prevent saddling. Height measurements are taken with septal rotational movement and sutured securely. If anchorage of the septum to the anterior nasal spine is deficient, some posterior and cephalic movement may lead to a supratip depression and hump recurrence. In Septum Pyramidal Adjustment and Repositioning (SPAR) concept and when possible, Dewes developed a strategy to retain a stripe of basal septum, especially at the anterior nasal spine, to stabilize more easily and effectively. ${ }^{32,33}$

\section{Pyramid Lateralization}

Apart from hump recurrence, pyramid lateralization is probably the most common reason for revision. With good septal stabilization, in deviated noses, the longer wall is approached by LDO and the shorter with PDO. Alternative is LDO
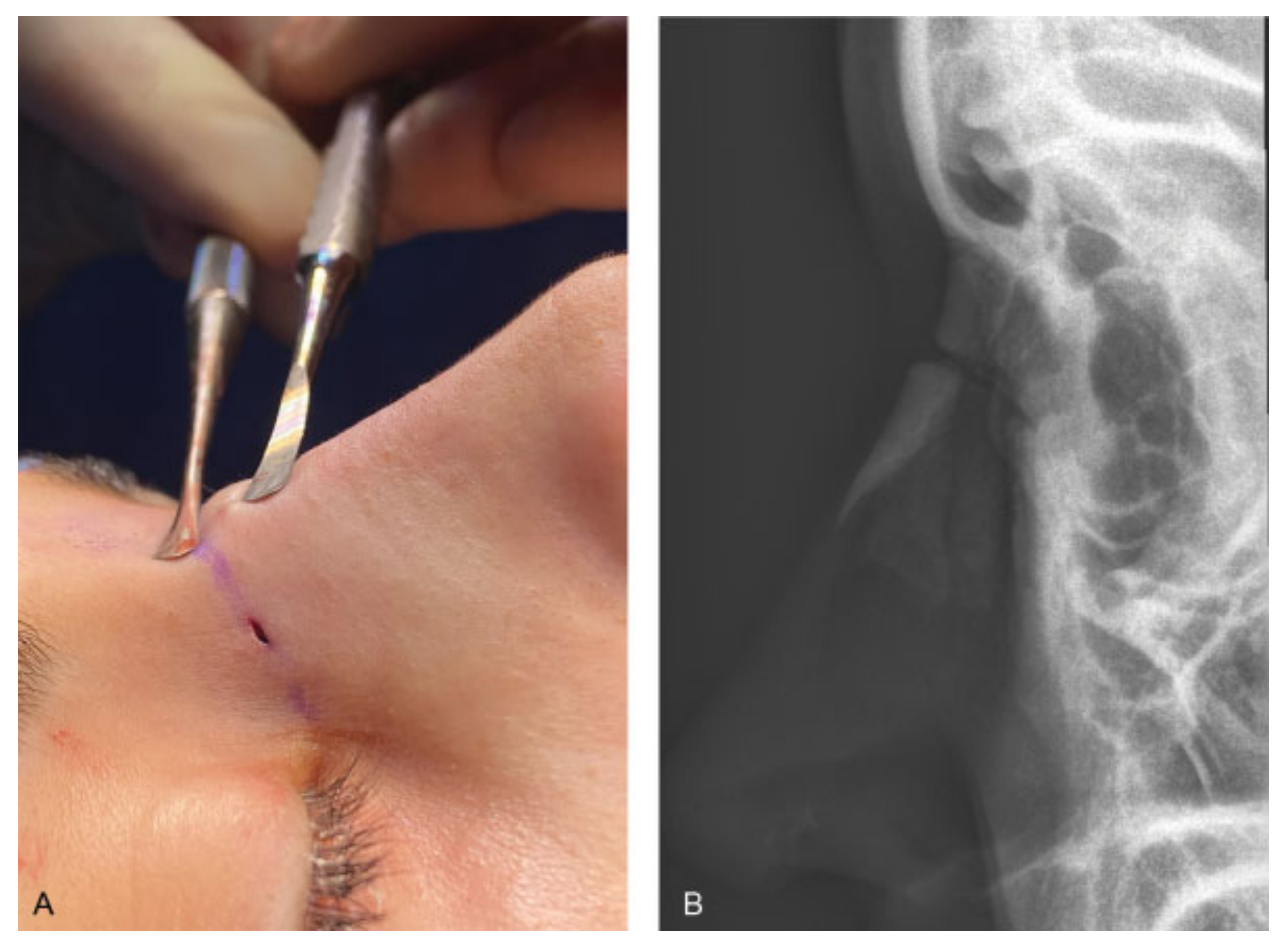

Fig. 15 (A) The bony elevation created by the step-up technique. (B) Postoperative X-ray showing the step-up. 


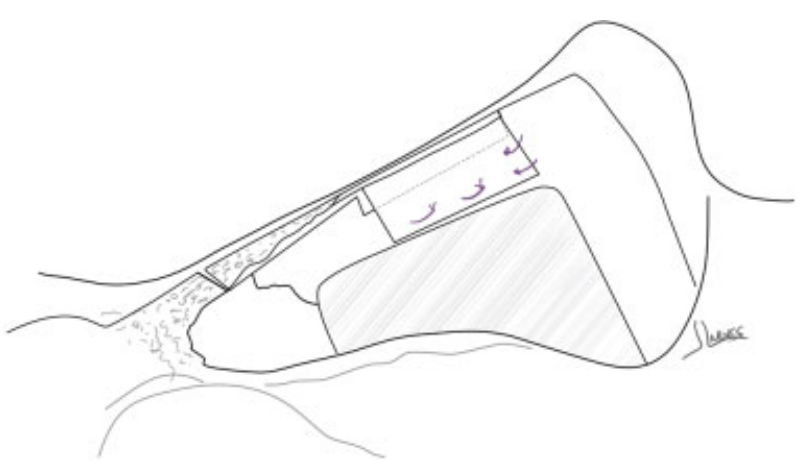

Fig. 16 The lateral Tetris concept in deviated pyramids. When performing the procedure in deviated pyramids it is indicated that there would be no slot creation below the Tetris block (red line) and consequently no trapezoid resection as seen in previous demonstrations; the block will suture to the stable septum in the opposite site of the deviation for compensation. The grey grid represents the septal harvesting leaving a stable $L$-shaped septum after suturing the Tetris block.

technique allowing pyramid movement on both sides facilitating repositioning.

The low approach techniques (Cottle or SPAR) are the best indications for the treatment of deviated noses that need a complete septoplasty and a septal repositioning. The "lateral Tetris," ${ }^{29,30}$ which is a partial intermediate approach, overlaps the free septal cartilage at the opposite side of the deviation and compensates for smaller pyramid lateralization (-Fig. 16).

The worst scenario is converting a straight nose to a deviated pyramid. To avoid this, lateral wall should be free to move but the septum should be fixed firmly. Even in a straight structure conflict at the inner concavity of the vault can be seen at the septal wall. When the triangular piece of the septal wall is removed below the bony vault, it is common to see a residual septum coming from the concave roof, which is sometimes difficult to remove completely, and especially at the perpendicular ethmoidal plate. During the deprojection maneuver, this residual septum may assume a side-to-side position with the basal septum and deviates the nasal axis, especially at the radix level. For this reason, accurate reduction and fixation is important. A definite advantage of designing the Tetris block is the two-axis stabilization in straight septum, by blocking cephalic-caudal and anterior-posterior vector movements (-Fig. 17).

\section{Broad Pyramids and Mid Third Broadening}

Broad nasal pyramids and irregular dorsum surfaces are generally contraindications for dorsal preservation rhinoplasty. Broad cartilaginous vault particularly at its cephalic portion is a prime example.

\section{Bony Pyramid}

Having the pyramid set in its new intended position, refinements such as sculpting and thinning the pyramidal bone are done. A partial paramedian osteotomy (using an ultrasonic device) may be placed at the dorsal aesthetic lines to reduce the width (-Fig. 8).

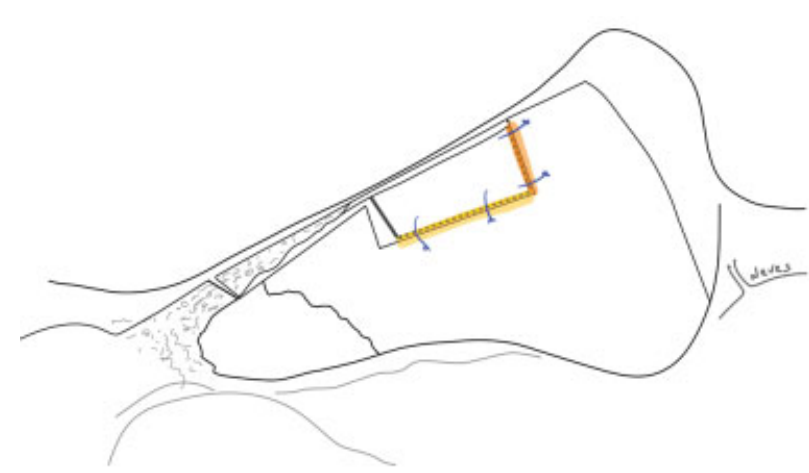

Fig. 17 Two axis stabilization. The posterior border of the block (yellow) avoids oblique axial-coronal tilting (mainly axial). The caudal border of the block (orange) avoids oblique coronal-axial tilting (mainly coronal).

\section{Cartilaginous Pyramid}

During the deprojection maneuver, the cartilaginous mid third tends to widen. This can be advantageous as it opens the internal valve but has aesthetic drawback. It can be avoided dissecting the LKA and liberating the lateral wall for a free anterior to posterior movement of the ULC. Care must be taken to avoid central key stone area disarticulation.

\section{Discussion}

We analyzed 100 consecutive cases operated with dorsal preservation rhinoplasty following the surgical concepts previously described to evaluate eventual drawbacks. The following were inclusion criteria: primary rhinoplasty, full dorsal preservation concepts, no grafts over the dorsum, and minimum 3 months follow-up. Postoperative analyses were restricted to nasal dorsum only. Tip issues and revisions were excluded.

Three different approaches were used in the patients: (1) Tetris concept-in straight tension and kyphotic noses (51 patients), (2) Lateral Tetris, in minor pyramid lateralization with convex profile (30 patients), (3) modified SPAR B (low strip approach), for severe lateralization and more extensive septoplasties (19 patients). The results are tabulated below (-Table 2)

When assessing drawbacks on profile and frontal views, strict criteria were followed to avoid bias (such as a mild hump). In the profile view, any deviation from the ideal straight profile line was considered a drawback (even with minimal or no impact on aesthetic outcome) and in the frontal view minimal pyramidal deviation was considered a drawback.

Residual humps and hump recurrences were seen in seven patients (36.9\%) in the Modified SPAR B, 3 with a minimal convexity of the profile, three with acceptable smooth convexity and only one patient (5.2\%) with indication for revision. In the Tetris approach no residual global convexity was identified but four patients (4.9\%) showed a residual bony hump, due to the stability of the cartilaginous vault based on the subrhinion suture but can show a kyphotic bone that was not ideally reshaped.

Radix steps were not relevant in the Tetris concept (one case, $1.2 \%$ ) but were palpable and slightly visible in $15.7 \%$ 
Table 2 Dorsal preservation rhinoplasty approaches and eventual drawbacks

\begin{tabular}{|c|c|c|c|c|c|c|c|c|}
\hline \multirow[b]{2}{*}{ Number of surgeries } & \multicolumn{2}{|c|}{ Tetris concept } & \multicolumn{2}{|c|}{$\begin{array}{l}\text { Lateral Tetris } \\
\text { concept }\end{array}$} & \multicolumn{2}{|c|}{ Modified SPAR B } & \multicolumn{2}{|c|}{ Total } \\
\hline & 51 & & 30 & & 19 & & 100 & \\
\hline Residual hump & 5 & $9.8 \%$ & 1 & $3.3 \%$ & 4 & $21.0 \%$ & 10 & $10.0 \%$ \\
\hline Rhinion & 0 & 0 & 0 & 0 & 3 & $15.7 \%$ & 3 & $3.0 \%$ \\
\hline Bony hump & 3 & $5.8 \%$ & 1 & $3.3 \%$ & 1 & $5.2 \%$ & 5 & $5.0 \%$ \\
\hline Cartilaginous hump & 2 & $3.9 \%$ & 0 & 0 & 0 & 0 & 2 & $2.0 \%$ \\
\hline Hump recurrence & 1 & $1.9 \%$ & 0 & 0 & 3 & $15.7 \%$ & 4 & $4.0 \%$ \\
\hline Rhinion depression & 3 & $5.8 \%$ & 1 & $3.3 \%$ & 0 & 0 & 4 & $4.0 \%$ \\
\hline Radix step & 0 & 0 & 1 & $3.3 \%$ & 3 & $15.7 \%$ & 4 & $4.0 \%$ \\
\hline Nasal infantilization & 0 & 0 & 0 & 0 & 1 & $5.2 \%$ & 1 & $1.0 \%$ \\
\hline Supratip saddling & 0 & 0 & 0 & 0 & 0 & 0 & 0 & 0 \\
\hline Pyramid lateralization & 1 & $1.9 \%$ & 2 & $6.7 \%$ & 5 & $26.3 \%$ & 8 & $8.0 \%$ \\
\hline Pyramid broadening & 0 & 0 & 2 & $6.7 \%$ & 1 & $5.2 \%$ & 3 & $3.0 \%$ \\
\hline Upper third & 0 & 0 & 2 & $6.7 \%$ & 1 & $5.2 \%$ & 3 & $3.0 \%$ \\
\hline Mid third & 0 & 0 & 0 & 0 & 0 & 0 & 0 & 0 \\
\hline Patients with any kind of remark & 7 & $13.7 \%$ & 4 & $13.3 \%$ & 9 & $47.3 \%$ & 20 & $20.0 \%$ \\
\hline Patients for revision & 2 & $3.9 \%$ & 1 & $3.3 \%$ & 1 & $5.2 \%$ & 4 & $4.0 \%$ \\
\hline
\end{tabular}

Abbreviation: SPAR, Septum Pyramidal Adjustment and Repositioning.

(three patients) in the Modified SPAR. When performing an extensive septoplasty, the perpendicular ethmoidal plate can lose stability leading to the lack of support of radix pillar, thought of promoting the radix step.

The same occurs when analyzing pyramid lateralization. The "lateral Tetris" was indicated in tilted noses, while the SPAR B was indicated in more complex cases with deviation. Five patients, (26.3\%) showed some degree of deviation from the central axis, even if all of them showed a great improvement of the initial condition. Four of these patients were very satisfied with the minimal lateralization; one patient was indicated for surgery. The same patient also had hump recurrency, being part of the seven previously discussed patients with hump recurrence. One case of the Tetris concept performed in a straight nose developed a full pyramid deviation. New osteotomies and repositioning were performed in a very conservative fashion.

Nasal mid third broadening was never an issue, justified by the constant realization of an LKA disarticulation.

Summing up, we identified $20 \%$ of the patients with some kind of remarks which, although apparently high due to our rigid criteria, are subjective. Nevertheless, only four (4\%) needed revision: one $(1 \%)$ for reshaping a residual bony hump after Tetris concept, two (2\%) to correct pyramid lateralization after Tetris and lateral Tetris concept, and another (1\%) to correct a pyramid lateralization with a hump recurrence after performing a modified SPAR B.

\section{Conclusion}

In appropriate patients dorsal preservation rhinoplasty is a safe and a natural operation to deproject the nasal pyramid.
Correct patient selection remains the first step to avoid drawbacks and complications. Even with this approach, several stigmata and drawbacks may be seen. It therefore follows that, each must be anticipated, analyzed, and controlled. The best way to approach the nasal pyramid is by segments, interpreting their characteristics and solutions, to achieve a predictable, accurate, and aesthetically pleasing result.

Conflict of Interest

None declared.

\section{References}

1 Goodale JL. A new method for the operative correction of exaggerated Roman nose. Boston Med Surg J 1899;140:112

2 Lothrop OA. An operation for correcting the aquiline nasal deformity; the use of new instrument; report of a case. Boston Med Surg J 1914; 170:835-837

3 Cottle MH, Loring RM. Corrective surgery of the external nasal pyramid and the nasal septum for restoration of normal physiology. Ill Med J 1946;90:119-135

4 Cottle MH. Nasal roof repair and hump removal. AMA Arch Otolaryngol 1954;60(04):408-414

5 Huizing EH. Push-down of the external nasal pyramid by resection of wedges. Rhinology 1975;13(04):185-190

6 Gola R. Conservative rhinoplasty. Ann Chir Plast Esthet 1994;38 (03):239-252

7 Gola R. La Rhinoplastie Fonctionnelle et Esthétique. Paris: Springer-Verlag; 2000:360

8 Gola R. Functional and esthetic rhinoplasty. Aesthetic Plast Surg 2003;27(05):390-396

9 Joseph J. The classic reprint: nasal reductions. Plast Reconstr Surg 1971;47(01):79-83

10 Joseph J. Beiträge zur Rhinoplastik. Berl Klin Wochenschrift. $1907 ; 16: 470-472$ 
11 Sebileau P, Dufourmentel L. Correction chirurgicale des difformités congénitales et acquises de la pyramide nasale. Paris: Arnette; 1926:104-105

12 Maurel G. Chirurgie maxilla-faciale. Paris: Le François; 1940: 1127-1133

13 Skoog T. A method of hump reduction in rhinoplasty. A technique for preservation of the nasal roof. Arch Otolaryngol 1966;83(03): 283-287

14 Huffman WC, Lierle DM. Rhinoplastic surgery: some general considerations. J Iowa State Med Soc 1948;38(10):443-447

15 Willemont J. Plastic surgery and otorhinolaryngology. Acta Otorhinolaryngol Belg 1967;21:463-732

16 Wayoff M, Perrin C. Global mobilization of the nasal pyramid according to Cottle's technic: its possibilities in functional nose surgery. Acta Otorhinolaryngol Belg 1968;22(06):675-680

17 Hinderer KH. Fundamentals of anatomy and surgery of the nose. Ann Arbor, MI: Aesculapius Publishing Co.; 1971

18 Drumheller GW. The push down operation and septal surgery. Boston: Little, Brown, and Company; 1973

19 Barelli PA. Long term evaluation of "push down" procedures. Rhinology 1975;13(01):25-32

20 Pirsig W, Königs D. Wedge resection in rhinosurgery: a review of the literature and long-term results in a hundred cases. Rhinology 1988;26(02):77-88

21 Saban Y, Daniel RK, Polselli R, Trapasso M, Palhazi P. Dorsal preservation: the push down technique reassessed. Aesthet Surg J 2018;38(02):117-131

22 Saban Y, Braccini F, Polselli R. Morphodynamic anatomy of the nose. In: Saban Y, Braccini F, Polselli R, Micheli-Pellegrini V. Rhinoplasties. Paris: The Monographs of CCA Group no. 32; 2002:25-32
23 Ishida J, Ishida LC, Ishida LH, Vieira JC, Ferreira MC. Treatment of the nasal hump with preservation of the cartilaginous framework. Plast Reconstr Surg 1999;103(06):1729-1733, discussion 1734-1735

24 Daniel RK. The preservation rhinoplasty: a new rhinoplasty revolution. Aesthet Surg J 2018;38(02):228-229

25 Montes-Bracchini JJ. Nasal profile hump reduction using the letdown technique. Facial Plast Surg 2019;35(05):486-491

26 Neves JC, Arancibia Tagle D, Dewes W, et al. The split preservation rhinoplasty: "the Vitruvian Man split maneuver. Eur J Plast Surg 2020;43:323-333

27 Goksel A, Saban Y. Open piezo preservation rhinoplasty: a case report of the new rhinoplasty approach. Facial Plast Surg 2019;35(01):113-118

28 Goksel A. Piezo assisted let down rhinoplasty. In: Daniel RK, Palhazi P, Saban Y, Cakir B, (eds). Preservation Rhinoplasty, 3rd edn. Istanbul: Septum Publisher; 2020:216-241

29 Neves JC, Arancibia Tagle D, Dewes W, Ferraz M. The segmental preservation rhinoplasty: The split Tetris concept. Facial Plast Surg 2020 (Epub ahead of print). doi: 10.1055/s-0040-1714672

30 Neves JC, Tagle DA, Dewes W, Ferraz M. A segmental approach in dorsal preservation rhinoplasty: the Tetris concept. Facial Plast Surg Clin North Am 2021;29(01):85-99

31 Lazovic GD, Daniel RK, Janosevic LB, Kosanovic RM, Colic MM, Kosins AM. Rhinoplasty: the nasal bones-anatomy and analysis. Aesthet Surg J 2015;35(03):255-263

32 Ferraz M, Zappelini C, Carvalho G, Guimarães A, Chone C, Dewes W. Cirurgia conservadora do dorso nasal-a filosofia do reposicionamento e ajuste do septo piramidal (S.P.A.R.). Rev. Bras. Cir. Cabeça Pescoço 2013;42(03):124-130

33 Dewes W. Rinoplastia conservadora: Septo Piramidal, Ajuste e Reposicionamento De A. Tratado de Otorrinolaringologia e Cirurgia Cérvicofacial Da ABORL-CCF. Philadelphia, PA: Elsevier; 2017:149 